\title{
A panel of antibodies to determine site of origin and malignancy in smooth muscle tumors
}

\author{
Cheng-Han Lee ${ }^{1}$, Dmitry A Turbin ${ }^{1}$, Y-C Vanessa Sung ${ }^{1}$, Inigo Espinosa ${ }^{2}$,
} Kelli Montgomery ${ }^{3}$, Matt van de Rijn ${ }^{3}$ and C Blake Gilks ${ }^{1}$

${ }^{1}$ Department of Pathology and Laboratory Medicine, University of British Columbia, Vancouver General Hospital, Vancouver, BC, Canada; ${ }^{2}$ Department of Pathology, Hospital de la Santa Creu i Sant Pau, Barcelona, Spain and ${ }^{3}$ Department of Pathology, Stanford University Medical Center, Stanford, CA, USA

\begin{abstract}
Leiomyosarcomas are malignant smooth muscle tumors that occur most commonly in the gynecologic tract and soft tissue. There are different diagnostic criteria of malignancy for smooth muscle tumors arising at gynecologic and soft tissue sites and they may be managed differently but determining the primary site of a smooth muscle tumor can be difficult in some cases. In addition, the distinction between malignant and benign gynecologic tract smooth muscle tumors on morphologic grounds can be challenging. Using a series of tissue microarrays that contain 245 cases of leiomyosarcomas (102 gynecologic) with survival data, and 49 cases of uterine leiomyoma, we examined the ability of selected immune-markers (estrogen receptor (ER) and WT1) to distinguish between leiomyosarcomas of gynecologic and nongynecologic origin. In addition, we examined whether immunostains for p16, p53 and Ki-67 could distinguish between malignant and benign gynecologic smooth muscle tumors. ER nuclear positivity was observed in 3 and $50 \%$ of the nongynecologic and gynecologic leiomyosarcomas, respectively $(P<0.001)$. Nuclear WT1 positivity was seen in 0 and $8 \%$ of the nongynecologic and gynecologic leiomyosarcomas, respectively $(P<0.001) .87 \%$ of primary gynecologic leiomyosarcomas and $2 \%$ of uterine leiomyomas showed diffuse $(\geqslant 50 \%$ of cells) p16 staining $(P<0.001) .23 \%$ of gynecologic leiomyosarcomas showed p53 immunopositivity ( $\geqslant 50 \%$ of cells) whereas none of the leiomyomas were positive for p53 $(P<0.001) .65 \%$ of the gynecologic leiomyosarcomas and $0 \%$ of the leiomyomas exhibited $>10 \% \mathrm{Ki}-67$ proliferation index $(P<0.001)$. Diffuse p16 and p53 immunopositivity and high Ki-67 proliferation index, singly or in combination, yielded an overall sensitivity of $92 \%$ and specificity of $98 \%$ for distinguishing between gynecologic leiomyosarcomas and leiomyomas and can be used as indicators of malignancy for gynecologic smooth muscle tumors. Although ER positivity can be used to support the gynecologic origin of a leiomyosarcomas, nuclear WT1 immunostaining is of little use.

Modern Pathology (2009) 22, 1519-1531; doi:10.1038/modpathol.2009.122; published online 4 September 2009
\end{abstract}

Keywords: leiomyosarcoma; uterine leiomyoma; p16; p53; Ki-67; ER

Leiomyosarcomas are malignant mesenchymal tumors that can occur in the gynecologic tract, soft tissue compartment or visceral sites. Leiomyosarcomas can be broadly grouped into gynecologic type and nongynecologic type based on their anatomic site of origin. Despite the fact that gynecologic and

Correspondence: Professor CB Gilks, MD, Department of Pathology and Laboratory Medicine, University of British Columbia, Vancouver General Hospital, 910 West 10th Avenue, Vancouver, BC V5Z 1M9, Canada.

E-mail: Blake.Gilks@vch.ca

Received 26 May 2009; revised 8 July 2009; accepted 21 July 2009; published online 4 September 2009 nongynecologic smooth muscle tumors are derived from similar appearing cells, there are significant differences in the criteria used to predict behavior. For example, it has long been recognized that benign behaving smooth muscle tumors of the gynecologic tract can exhibit increased mitotic activity whereas the presence of similar mitotic activity in either deep or superficial soft tissue smooth muscle tumors would lead to an unequivocal diagnosis of leiomyosarcoma. ${ }^{1}$ Clinically, these two groups of malignancies are managed differently by different oncologic disciplines. ${ }^{2,3}$ Therefore adequate determination of gynecologic versus nongynecologic origin is crucial but can be problematic, especially in tumors that are 
large and located in the pelvis. The majority of uterine leiomyomas and a variable percentage (25$70 \%$ ) of uterine leiomyosarcomas express estrogen receptor (ER) and/or progesterone receptor (PR). ${ }^{4-11}$ This suggests that at least a subset of uterine leiomyosarcomas may originate from ER/PR expressing myometrial smooth muscle cells. ${ }^{12}$ In contrast, soft tissue and cutaneous leiomyosarcomas are believed to be derived from vascular smooth muscle and pilar smooth muscle, respectively, both of which lack intrinsic ER/PR expression. ER and WT1 are proposed immunomarkers for identification of primary site of leiomyosarcoma, but their utility has not been assessed in a large series of cases $^{4-11,13-15}$ with the largest series containing 38 cases.

Another issue of concern, especially for tumors arising in the gynecologic tract, is that it can be quite challenging to determine whether a smooth muscle tumor will behave in a benign or malignant manner. ${ }^{16}$ Significant mitotic activity, focal degenerative cellular atypia and/or hyaline necrosis can all be present in benign uterine leiomyoma. This can be particularly difficult when only a limited biopsy tissue sample is available for assessment. Several immunomarkers, most notably p16, p53 and Ki-67, have been examined previously as aids to distinguish between gynecologic leiomyosarcomas and leiomyomas. ${ }^{7,11,17-23}$ However, only small numbers of cases ( $<50$ cases) have been examined in each study, typically with a single marker. In addition, the appropriate diagnostic cut-points for use of these immunomarkers have not been critically evaluated.

In this study, we used a panel of antibodies to address two issues that complicate the diagnosis of smooth muscle tumors and determined cut-points for each marker. First, we examined the utility of ER and WT1 immunomarkers in differentiating between gynecologic and nongynecologic leiomyosarcomas, using a series of 245 leiomyosarcomas. We also assessed the utility of p16, p53 and Ki-67 immunomarkers, alone or in combination, as adjuncts to predict clinical behavior in gynecologic smooth muscle tumors, as tested on 90 primary gynecologic leiomyosarcomas and 49 uterine leiomyomas.

\section{Materials and methods}

\section{Case Selection and Tissue Microarray Construction}

Paraffin-embedded samples of 245 leiomyosarcomas from 245 different patients were collected from several hospitals and laboratories across the United States, Canada and the Netherlands (Table 1). A total of 149 cases were used previously in studies determining prognostic significance of macrophages $^{24}$ and CSF1-associated genes in leiomyosarcomas. ${ }^{25}$ Of the 245 leiomyosarcoma tumor samples, 195 were biopsy or resection specimens of the primary tumor, whereas the remaining 50 cases represented biopsy or resection specimens from recurrent (local or distant) disease. Clinical characteristics were available for 221 cases and diseasespecific survival (minimum follow-up of 6 months) were available for 74 cases of gynecologic leiomyosarcomas and 64 cases of nongynecologic leiomyosarcomas. For the primary tumors, none of these patients received neoadjuvant treatment (chemotherapy and/or radiotherapy). All of the cases were centrally reviewed (CHL, IE) and the diagnosis of leiomyosarcoma was confirmed based on histologic and immunohistochemical evaluation as described previously. ${ }^{24}$ The tumor cell grade was assigned based on the FNCLCC (Fédération Nationale des Centres de Lutte Contre le Cancer) grading system for all leiomyosarcomas. A total of 49 cases of uterine leiomyoma were also obtained from Vancouver General Hospital (Vancouver, BC, Canada) for comparison. The studies were performed with the approval from the institutional review boards at Vancouver General Hospital and Stanford University Medical Center.

Tissue microarrays were constructed as described previously using a manual tissue arrayer (Beecher Instruments, Silver Spring, MD, USA). ${ }^{24}$ For each leiomyosarcoma or uterine leiomyoma, a block with more cellular proliferative region of the tumor was chosen; scarred, hyalinized, myxoid or hypocellular areas were avoided. The final tissue microarrays were constructed with one $0.6 \mathrm{~mm}$ tissue core and duplicate $0.6 \mathrm{~mm}$ tissue cores for individual cases of leiomyosarcoma and uterine leiomyoma, respectively.

\section{Immunohistochemical Staining and Evaluation}

Antibodies that target ER, WT1, p16, p53 and Ki-67 were used in the current study. The details of the primary antibodies and the dilutions used are shown in Table 1. Antigen retrieval was performed using CC1 antigen retrieval buffer (Ventana Medical Systems, Tucson, AZ, USA) for all sections.

Table 1 List of the primary antibodies

\begin{tabular}{lll}
\hline ER & Anti-ER rabbit monoclonal antibody (clone SP1). Lab Vision, Fremont, CA, USA & $1: 50$ \\
WT1 & Anti-WT1 mouse monoclonal antibody (clone 6F-H2). Dako, Carpinteria, CA, USA & $1: 100$ \\
p16 & Anti-p16 & INK4a mouse monoclonal antibody (clone 16P04). Cell Marque, Hot Springs, AR, USA \\
p53 & Anti-p53 mouse monoclonal antibody (clone DO-7). Dako, Carpinteria, CA, USA & $1: 20$ \\
Ki-67 & Anti-Ki-67 rabbit monoclonal antibody (clone SP6). Lab Vision, Fremont, CA, USA & $1: 400$ \\
& &
\end{tabular}


Following incubation with the primary antibodies, sections were stained on the Ventana automated slide stainer (NEXES) using the Ventana diaminobenzidine detection kit (Ventana Medical Systems). Ventana amplification kit (Ventana Medical Systems) was also used for ER immunohistochemistry. For ER, positivity was defined as any positive nuclear staining in $\geq 5 \%$ of tumor cells. For WT1, positive nuclear staining was defined as the presence of any nuclear staining in $\geq 5 \%$ of tumor cells and positive cytoplasmic staining was defined as the presence of any cytoplasmic staining in $\geq 5 \%$ of tumor cells. For p16, positivity was defined as any positive cytoplasmic and/or nuclear staining in $\geq 50 \%$ of tumor cells. For p53, positivity was defined as moderate to strong positive nuclear staining in $\geq 50 \%$ of tumor cells. Ki-67 staining was evaluated both visually (CHL) and by automated morphometric analysis. For visual pathologist assessment, the percentage of positive nuclear staining in the tumor cells is quantified by visual estimation to the nearest $1 \%$ based on assessment of 100 tumor cell nuclei for each core. An average percentage was derived if duplicate cores are present for a given case.

\section{Automated Ki-67 Nuclear Staining Morphometric Analysis}

Details of the morphometric scoring were described previously. ${ }^{26}$ Briefly, digital images of sections from the TMA stained with Ki-67 were used for morphometric analyses, captured using a BLISS scanner (Bacus Laboratories, Lombard, IL, USA). The slides were scanned at $\times 200$ objective magnification. A custom-written nuclear stain analyzer software ImageJ (http://www.gpec.ubc.ca/index.php?content = software/imagej_plugin/index.php) was used, with the parameters set to optimally identify tumor nuclei. The number of nuclei in each core and its corresponding optical quality were registered. The output data showed the number of brown-stained nuclei (positive staining) and the number of bluestained nuclei (negative staining) for each core and the percentage of positively stained nuclei was calculated for each core.

\section{Statistical Analysis}

Scoring results were combined using Deconvoluter and TMA-Combiner programs (http://genome-www. stanford.edu/TMA/download.shtml). Kaplan-Meier analysis was used to show survival curves with logrank test to compare survival between two (or more) different groups (WinSTAT 2007). Unpaired Student's $t$-test was used for comparison of the demographic data wherever appropriate (Excel 2007). A $P$-value of less than 0.05 was considered significant.

\section{Results}

\section{Patient Demographics}

The patient demographic information is shown in Table 2. The 245 leiomyosarcoma samples consisted of 195 primary tumors (105 nongynecologic and 90 gynecologic leiomyosarcomas) and 50 recurrent tumors (38 nongynecologic and 12 gynecologic leiomyosarcomas). The overall age distribution was slightly younger for patients with gynecologic leiomyosarcomas compared to patients with nongynecologic leiomyosarcomas (49 vs 53 years, $P=0.16)$. Eighty-nine (90\%) of the primary gynecologic leiomyosarcomas in our series arose from the uterus whereas the remaining cases occurred in the vagina, adnexa and vulva. Eighty-six (82\%) nongynecologic leiomyosarcomas were based in

Table 2 Clinical and pathologic features of leiomyosarcomas

\begin{tabular}{|c|c|c|}
\hline & $\begin{array}{c}\text { Gynecologic } \\
\text { leiomyosarcoma }\end{array}$ & $\begin{array}{l}\text { Nongynecologic } \\
\text { leiomyosarcoma }\end{array}$ \\
\hline Total number of cases & 102 & 143 \\
\hline Primary & 90 & 105 \\
\hline Recurrence (local/distant) & 12 & 38 \\
\hline \multicolumn{3}{|l|}{ Age at time of diagnosis (years) } \\
\hline Average $^{\mathrm{a}}$ & 49 & 53 \\
\hline$\leq 40$ & 11 & 21 \\
\hline$\overline{41}-50$ & 39 & 34 \\
\hline $51-60$ & 40 & 30 \\
\hline$>60$ & 7 & 39 \\
\hline \multicolumn{3}{|l|}{$S e x^{b}$} \\
\hline Male & NA & 68 \\
\hline Female & 102 & 59 \\
\hline \multicolumn{3}{|l|}{ Primary tumors } \\
\hline \multicolumn{3}{|l|}{ Gynecologic Tract $(n=90)$} \\
\hline Uterus & 81 & NA \\
\hline Vagina & 3 & NA \\
\hline Adnexa & 4 & NA \\
\hline Vulva & 2 & NA \\
\hline \multicolumn{3}{|c|}{ Nongynecologic tract $(n=105)$} \\
\hline $\begin{array}{l}\text { Retroperitoneum/ } \\
\text { abdomen/pelvis }\end{array}$ & NA & 40 \\
\hline Soft tissue, limbs & NA & 29 \\
\hline Trunk & NA & 12 \\
\hline Genitourinary system & NA & 7 \\
\hline Thorax & NA & 5 \\
\hline Bone & NA & 5 \\
\hline Head and neck & NA & 3 \\
\hline Others & NA & 4 \\
\hline \multicolumn{3}{|l|}{ Recurrent tumors } \\
\hline Local recurrence & 7 & 22 \\
\hline Distant recurrence & 5 & 16 \\
\hline Lung & 4 & 12 \\
\hline Liver & & 2 \\
\hline Others & 1 & 2 \\
\hline
\end{tabular}

${ }^{\mathrm{a}}$ Age at time of diagnosis was not known for 5 gynecologic and 19 nongynecologic LMS cases.

${ }^{\mathrm{b}}$ Sex of the patient was not known for 18 nongynecologic LMS patients.

NA: not applicable. 
the deep soft tissue of the trunk, limb and body cavity, whereas the remainder occurred in sites such as the genitourinary tract, head and neck region, and bone. Of the 50 recurrent tumors, 29 were from local recurrences whereas 21 tumors represented metastatic tumors, with lung (16 of 21 metastatic tumors) being most common site of distant spread for both gynecologic and nongynecologic leiomyosarcomas. Of the 49 uterine leiomyomas, there were 2 cellular variants, 1 symplastic variant and 2 uterine leiomyomas showing focal infarction, whereas the remainder displayed usual histologic appearance.

\section{ER and WT1 Immunohistochemistry in Primary and} Recurrent Leiomyosarcomas and Uterine Leiomyomas

The results of ER and WT1 immunostaining for the 245 leiomyosarcomas and 49 uterine leiomyomas are depicted in Figure 1 with representative images showing the patterns of immunostaining in Figure 2. For gynecologic leiomyosarcomas, ER immunopositivity (defined as nuclear staining in $>5 \%$ of tumor cells) was present in 50\% (51 of 102 cases) of primary or recurrent gynecologic leiomyosarcomas, with 7 of the 12 recurrent tumors being positive for ER. In contrast, only $3 \%$ of nongynecologic leiomyosarcomas (4 of 140 scorable cases) showed positive ER nuclear staining $(P<0.001)$. Among the four ERpositive nongynecologic leiomyosarcoma cases, one occurred in the male genital region, one in the male rectal region and two in the female abdominal/pelvic region. For the last two cases, a primary gynecologic site could not be excluded with certainty. All 49 uterine leiomyomas showed positive ER staining.

For WT1, nuclear staining and cytoplasmic staining were assessed separately. Only 8\% (8 of 98 scorable cases) of gynecologic leiomyosarcomas showed weak-to-moderate nuclear staining whereas none of the nongynecologic leiomyosarcomas showed detectable nuclear staining $(P<0.001)$. The nuclear staining in the positive cases was predominantly weak in intensity (Figure 2). Positive cytoplasmic WT1 immunostaining (cytoplasmic staining in $>5 \%$ of tumor cells) was observed in $55 \%$ (54 of 98 scorable cases) of gynecologic leiomyosarcomas and $52 \%$ (68 of 131 scorable cases) of nongynecologic leiomyosarcomas $(P=0.60)$. In contrast to the intensity of nuclear staining, cytoplasmic staining was generally moderate to strong in intensity (Figure 2). 67\% (33 of 49 cases) and 90\% (44 of 49 cases) of uterine leiomyomas exhibited positive nuclear and cytoplasmic WT1 immunostaining, respectively. All of the cases that exhibited nuclear staining also showed cytoplasmic staining concurrently.

\section{p53, p16 and Ki-67 Immunohistochemistry in Primary Tumors}

The results of p53 and p16 immunostaining for the 195 primary leiomyosarcomas and 49 uterine
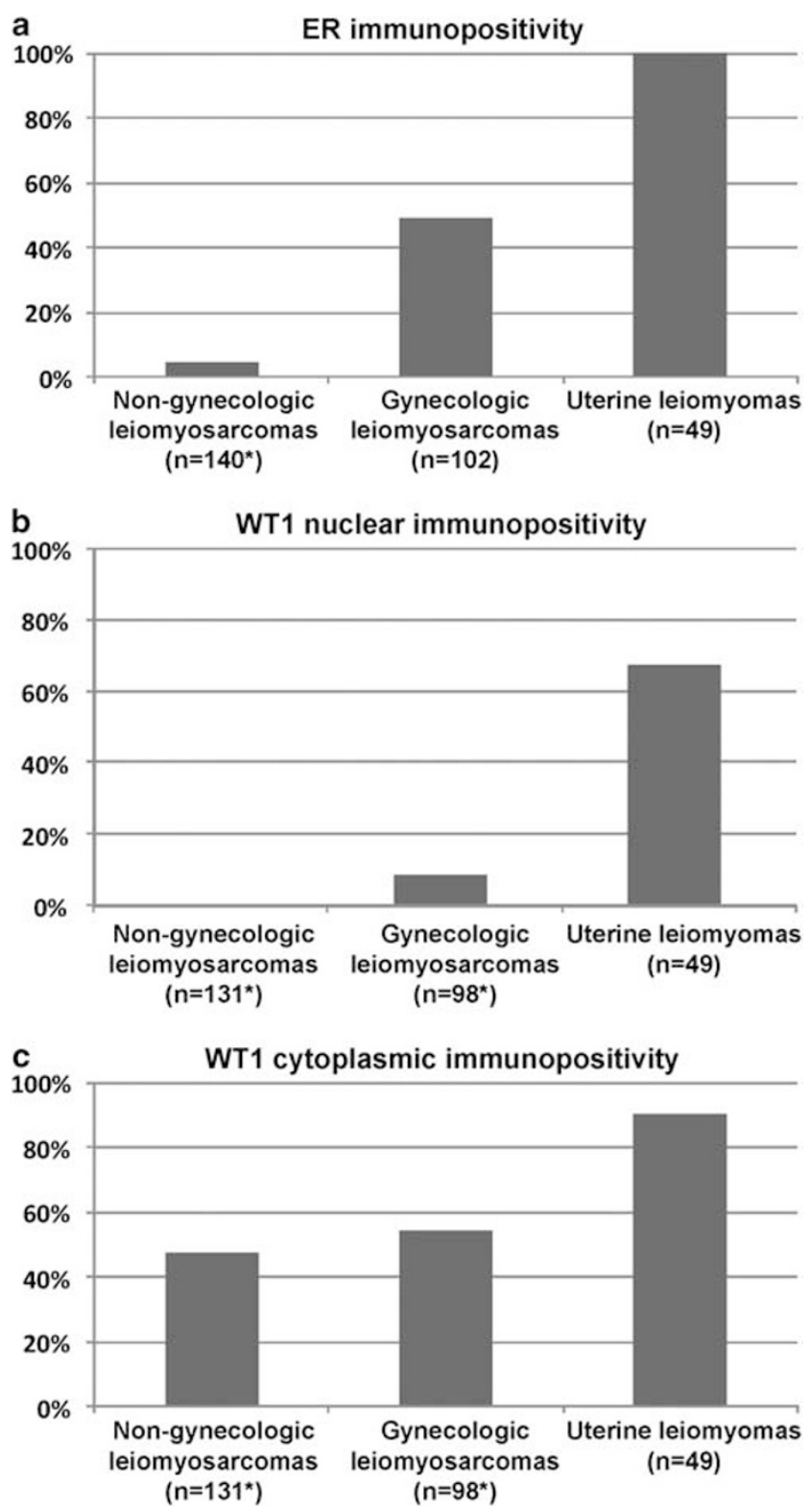

Figure 1 ER (a) and WT1 (b and c) immunostaining patterns in gynecologic and nongynecologic smooth muscle tumors ( ${ }^{*}$, number of scorable cases).

leiomyomas are depicted in Figure 3 with representative images showing the patterns of immunostaining in Figure 4. Positive p53 immunostaining (nuclear staining in at least $50 \%$ of tumor cells) was found in $23 \%$ (20 of 87 scorable cases) of primary gynecologic leiomyosarcomas whereas none of the 49 uterine leiomyomas showed positive p53 immunostaining $(P<0.001) .17 \% \quad(17$ of 96 scorable cases) of primary nongynecologic leiomyosarcomas showed positive p53 staining.

Positive p16 immunostaining (nuclear and/or cytoplasmic staining in $\geqslant 50 \%$ of tumor cells) was present in $87 \%$ (77 of 89 scorable cases) of primary gynecologic leiomyosarcomas and $2 \%$ ( 1 of 48 scorable cases) of uterine leiomyomas $(P<0.001)$. 


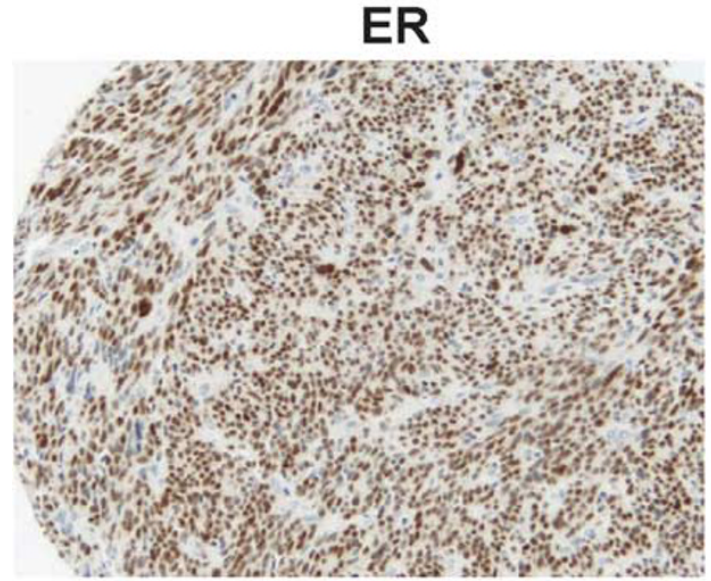

$E R(+)$ gynecologic leiomyosarcoma (no. 124)

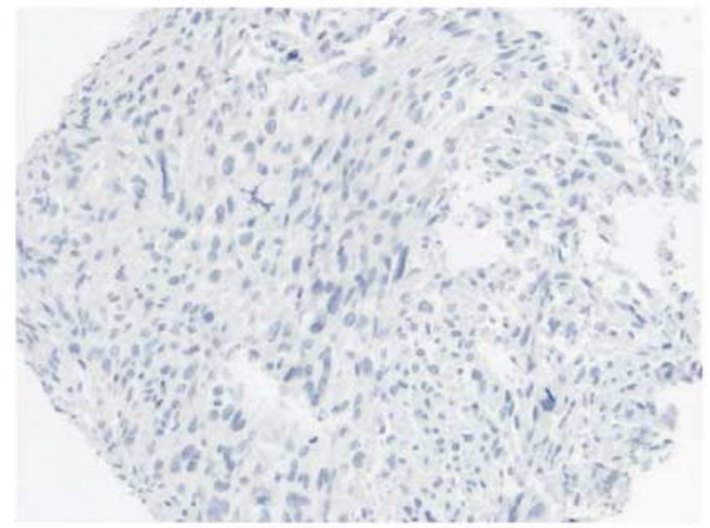

ER(-) Non-gynecologic leiomyosarcoma (no. 6601)

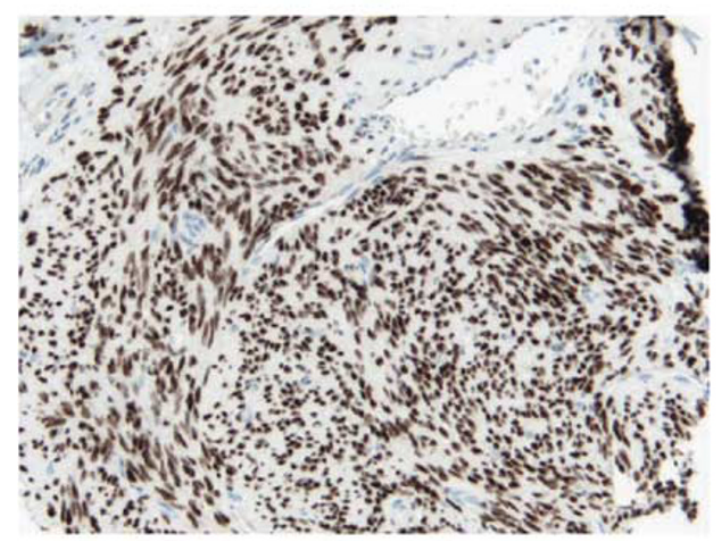

$E R(+)$ uterine leiomyoma

(no. 40)

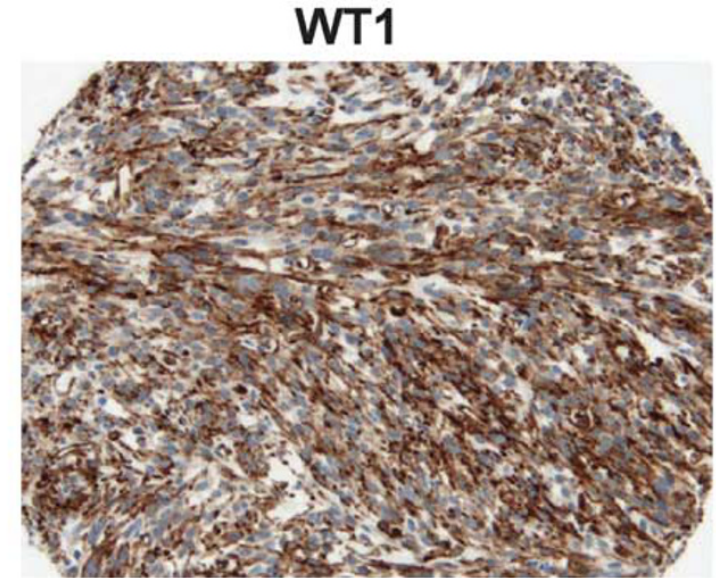

Cytoplasmic WT1(+)

gynecologic

leiomyosarcoma (no. 30)

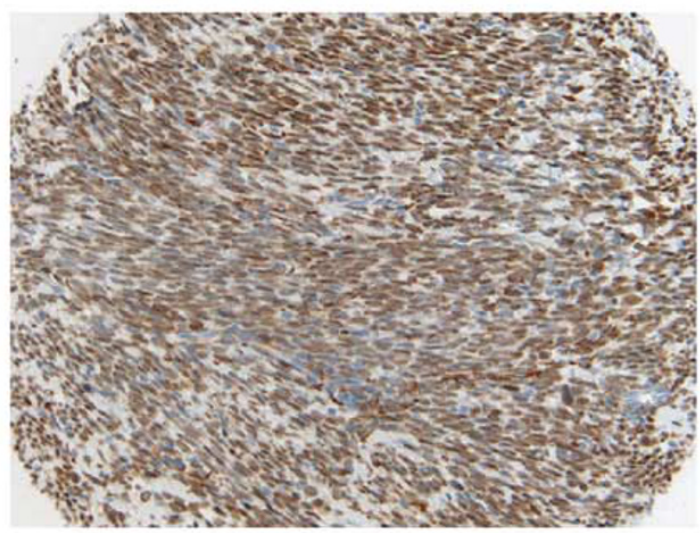

Nuclear WT1(+) gynecologic leiomyosarcoma (no. 59)

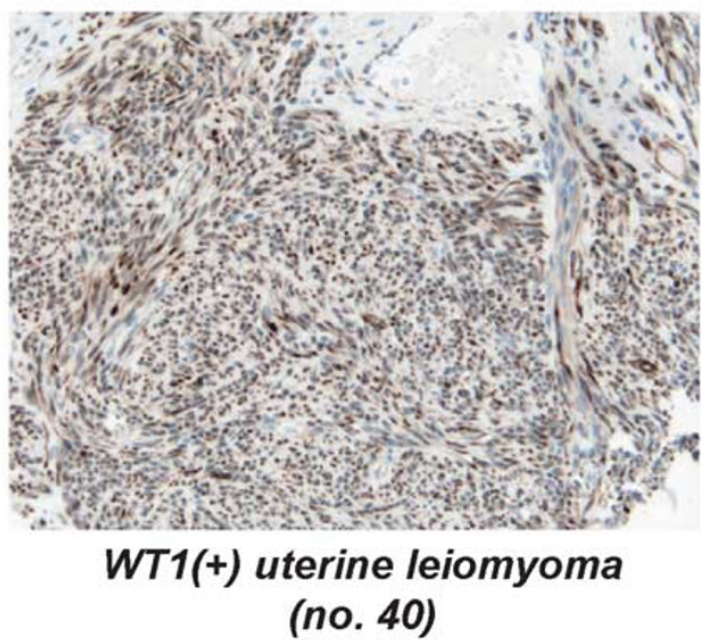

Figure 2 Representative images of ER and WT1 immunostaining in gynecologic and nongynecologic smooth muscle tumors.

The majority (46 of 48 scorable cases) of uterine leiomyomas showed only focal $(0-20 \%)$ p16 staining. A single case of uterine leiomyoma demon- strated weak cytoplasmic/nuclear p16 staining in $60 \%$ of tumor cells and it showed focal hyaline necrosis but no atypia or increased mitotic activity 

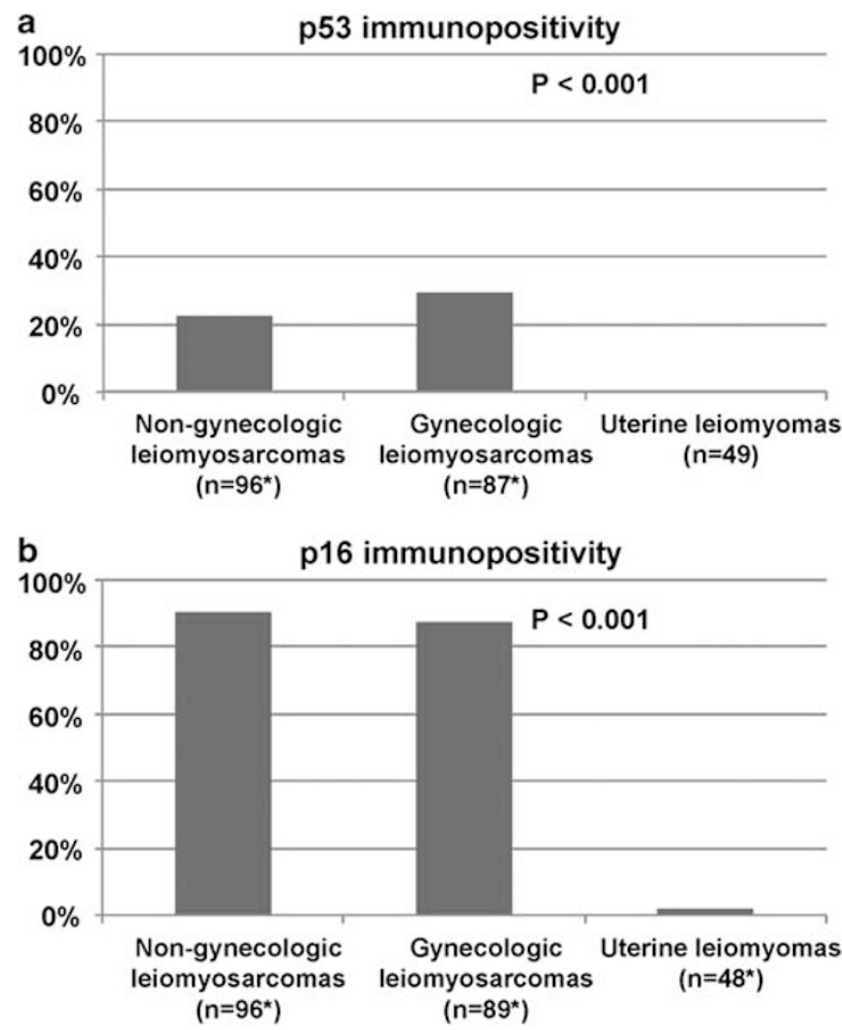

Figure 3 p53 (a) and p16 (b) immunostaining patterns in primary gynecologic and nongynecologic smooth muscle tumors ${ }^{*}$, number of scorable cases).

(Ki-67 staining of $1 \%$ by visual analysis and $0.55 \%$ by automated analysis). In comparison, $90 \%$ (86 of 96 scorable cases) of primary nongynecologic leiomyosarcomas were p16 positive. The positive cases displayed both nuclear and cytoplasmic immunostaining (Figure 4).

Ki-67 immunostaining was evaluated by both visual and automated methods as described in the Materials and methods section, with the results depicted in Figure 5. Based on the findings of prior studies $4,7,11,19,22,23,27-32$ a cutoff of $10 \%$ was used for both the visual and the automated analyses. The results of manual and automated analyses showed a correlation coefficient of 0.77 . Positive Ki-67 staining was observed in $64 \%$ (50 of 78 scorable cases) of primary gynecologic leiomyosarcomas and $0 \%$ (0 of 49 cases) of uterine leiomyomas by automated analysis $(P<0.001)$. Visual analysis yielded near-identical results with positive Ki-67 staining observed in $65 \%$ (51 of 78 scorable cases) of primary gynecologic leiomyosarcomas and $0 \%(0$ of 49 cases) of uterine leiomyomas $(P<0.001)$. A total of eight cases showed discrepant results between the automated and visual analyses with four cases assessed to be positive by visual method but not by automated method and four cases assessed to positive by automated method but not by the visual method. All the eight cases showed positive p16 immunostaining. By automated analy- sis, uterine leiomyomas have an average proliferative index of $1.6 \%$ (range from 0 to $8.3 \%$ ) and primary gynecologic leiomyosarcomas has an average of $23.4 \%$ (range from 0.1 to $86.1 \%$ ). The highest proliferation index displayed by any of the uterine variants or uterine leiomyomas with focal hyaline necrosis, was $3.1 \%$. For primary nongynecologic leiomyosarcomas, $67 \%$ (53 of 79 scorable cases) and $71 \%$ (56 of 79 scorable cases) were found to exhibit positive Ki-67 immunostaining by automated analysis and manual analysis, respectively.

The overall immunostaining results are illustrated graphically in Figure 6. Positivity with at least one of p16, p53 or Ki-67 immunomarkers was seen in 81/ $88(92 \%)$ of gynecologic leiomyosarcomas, compared to $1 / 48(2 \%)$ of uterine leiomyomas (sensitivity $92 \%$, specificity $98 \%$ ). Results of the current study are compared to previously published findings summarized in Table 3.

\section{Kaplan-Meier Survival Analysis of Immunostaining Results}

Disease-specific survival data, with a median follow-up period of 3 years, were available for 74 of 90 primary gynecologic leiomyosarcomas and 64 of 105 primary nongynecologic leiomyosarcomas. The Kaplan-Meier survival curves for gynecologic and nongynecologic leiomyosarcomas are depicted in Supplementary Figure $1(P=0.73)$. Nongynecologic leiomyosarcomas showed an estimated 5-year disease-specific survival of $70 \%$. In contrast, gynecologic leiomyosarcomas showed an estimated 5-year disease-specific survival of below $60 \%$ and the survival curve continues to exhibit a downward trend even toward the latter portion of the 5-year period. There was no significant difference in survival based on Ki-67 labeling index, comparing cases with greater than or less than $10 \%$ proliferation index, for either gynecologic leiomyosarcomas $(P=0.61) \quad$ or nongynecologic leiomyosarcomas $(P=0.23)$ (Supplementary Figure 1$)$. No significant association with outcome was found when the median of the Ki-67 proliferation index was used to subdivide the gynecologic or nongynecologic leiomyosarcomas (data not shown). For ER and p53, there were no significant associations observed between disease-specific survival and the immunostaining findings (Supplementary Figure 2).

\section{Discussion}

We have used the largest series of smooth muscle tumors examined to date to assess the utility of ER and WT1 in differentiating between gynecologic and nongynecologic leiomyosarcomas, and the utility of p16, p53 and Ki-67 in differentiating between gynecologic leiomyosarcomas and uterine leiomyomas. The findings confirm the results of previous 


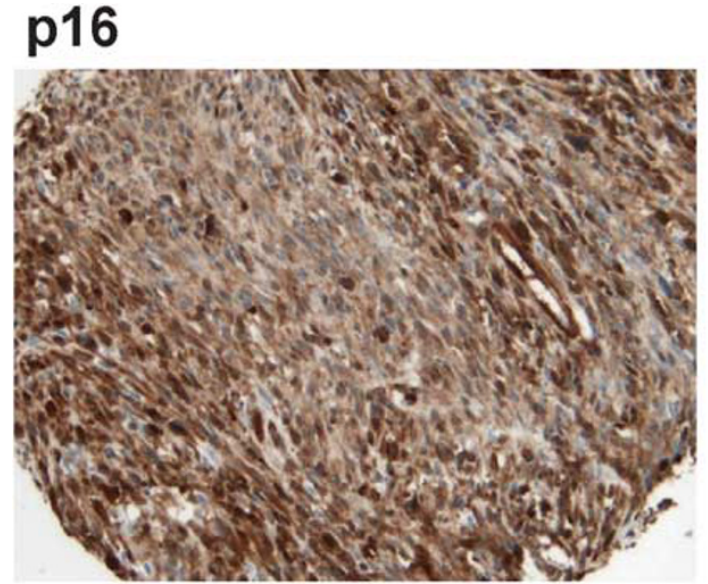

p16(+) gynecologic leiomyosarcoma (no. 53)

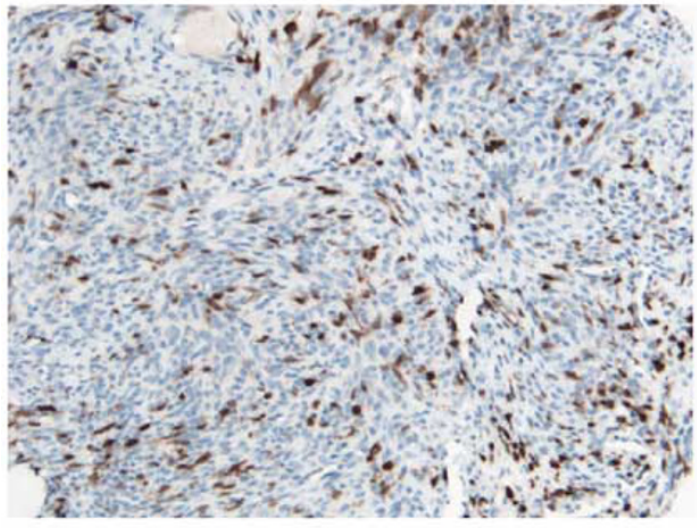

p16(-) uterine leiomyoma

(no. 9)

p53

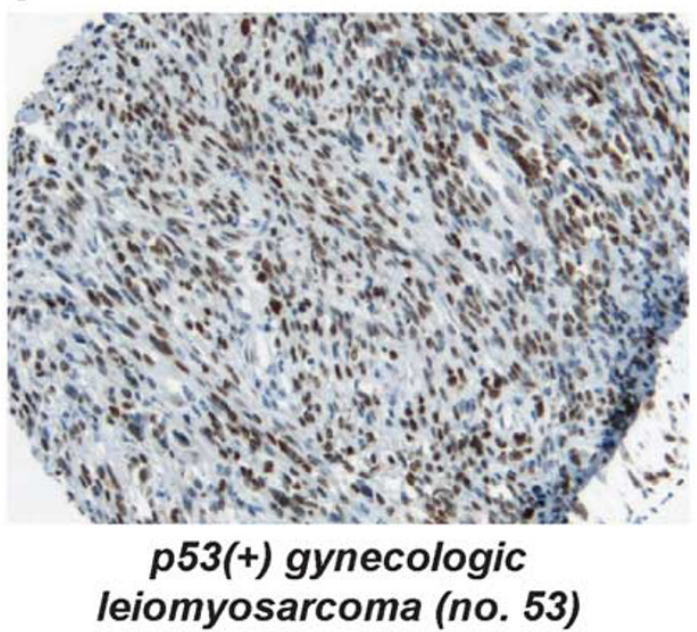

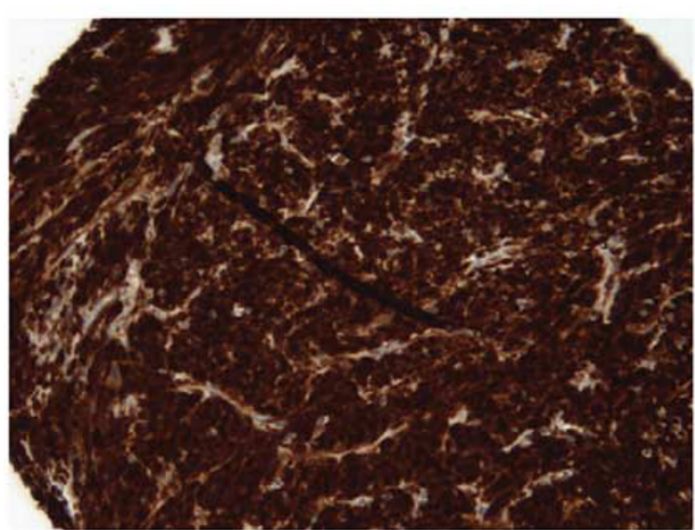

p16(+) gynecologic leiomyosarcoma (no. 124)

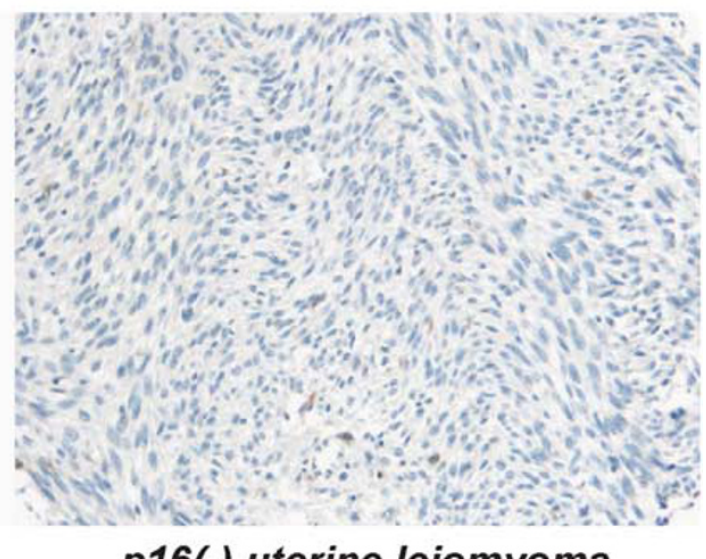

p16(-) uterine leiomyoma

(no. 38)

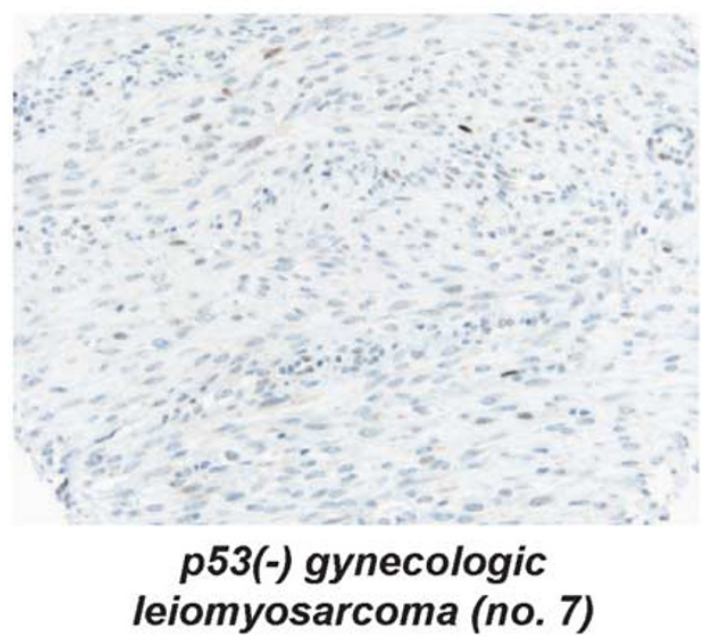

Figure 4 Representative images of p53 and p16 immunostaining in gynecologic and nongynecologic smooth muscle tumors.

studies and demonstrate the value of ER immunostaining in differentiating between leiomyosarcomas of gynecologic and nongynecologic origins and the utility of combined p16, p53 and Ki-67 panel in differentiating between malignant and benign gynecologic smooth muscle tumors. 


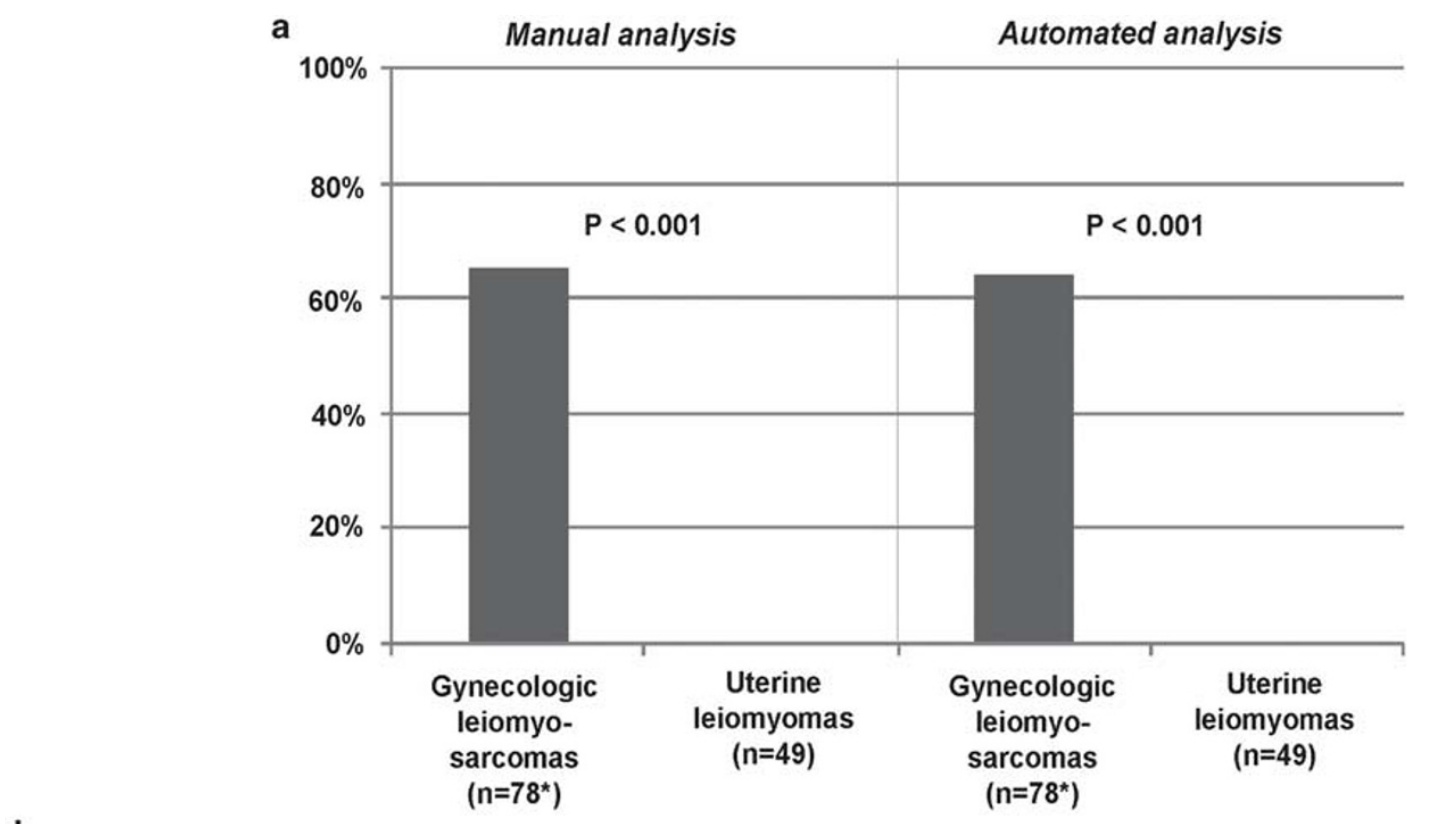

b

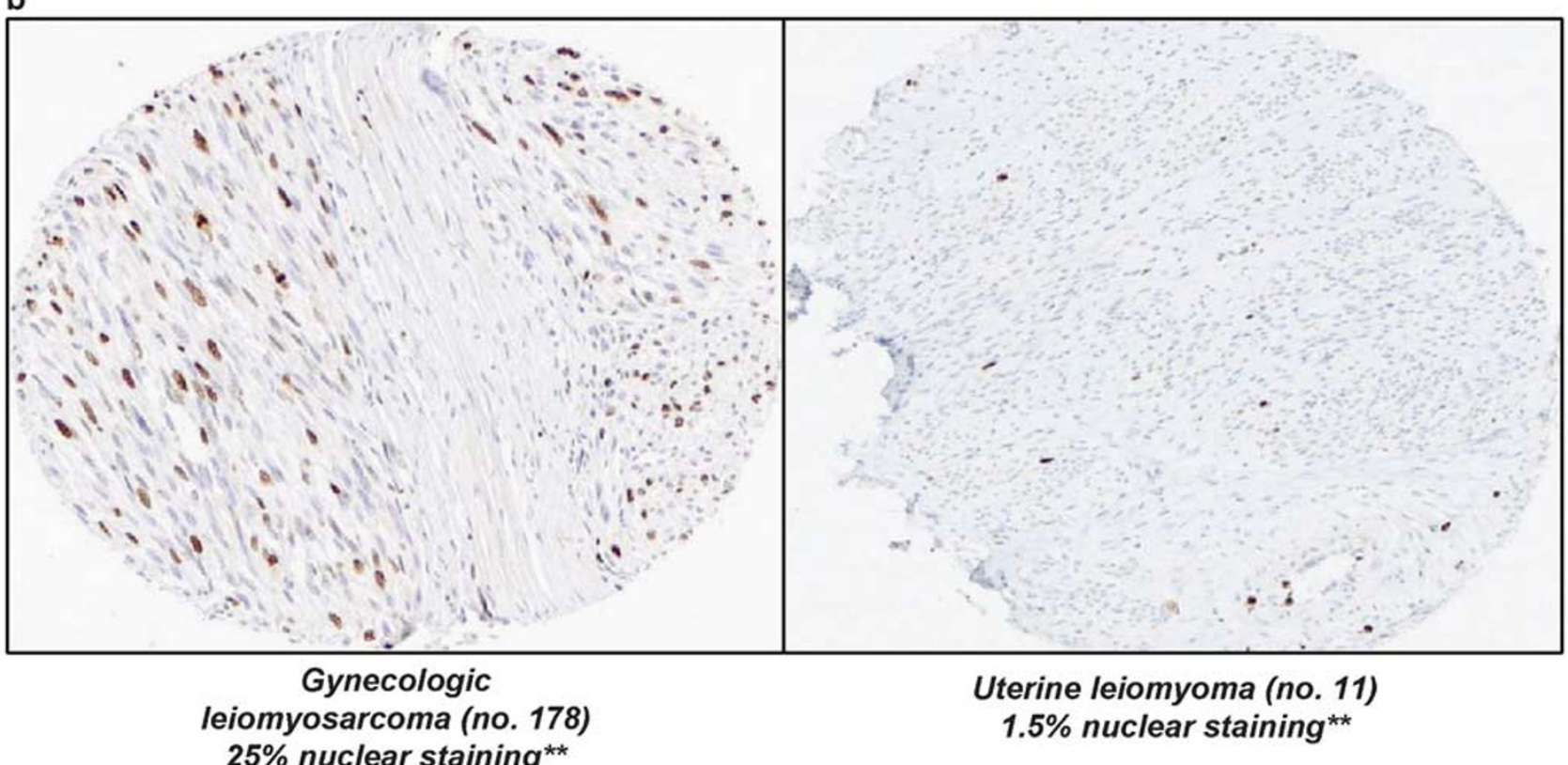

Figure 5 (a) Ki-67 immunostaining patterns in primary gynecologic and nongynecologic smooth muscle tumors (*, number of scorable cases). (b) Representative images of Ki-67 immunostaining results $\left({ }^{* *}\right.$, percent nuclear staining determined by automated morphometric analysis).

\section{Adjunct Markers to Differentiate between Leiomyosarcomas of Gynecologic and Nongynecologic Origin}

Two immunomarkers-ER and WT1-were examined for their utility in differentiating between leiomyosarcomas of gynecologic and nongynecologic origins. ER was found to be differentially expressed by gynecologic leiomyosarcomas (50\% ER positive) and nongynecologic leiomyosarcomas ( $3 \%$ ER positive), such that the presence of ER immunoreactivity in leiomyosarcoma from either primary or metastatic sites would strongly favor a gynecologic origin with a specificity of $94 \%$. The combined results of previous studies (for 129 cases) showed a very similar finding with $52 \%$ ER positivity in gynecologic leiomyosarcomas. ${ }^{4-11}$ Using a cutoff of $5 \%$ tumor cell nuclear staining, Rao et $a l^{10}$ reported ER positivity in $6 \%$ of nongynecologic leiomyosarcomas, a finding similar to our current observation. In contrast, Kelly et $a l^{8}$ used a lower cutoff of $1 \%$ and observed ER positivity in 4 of 16 (25\%) nongynecologic leiomyosarcomas, with 3 of the 4 positive cases from female 

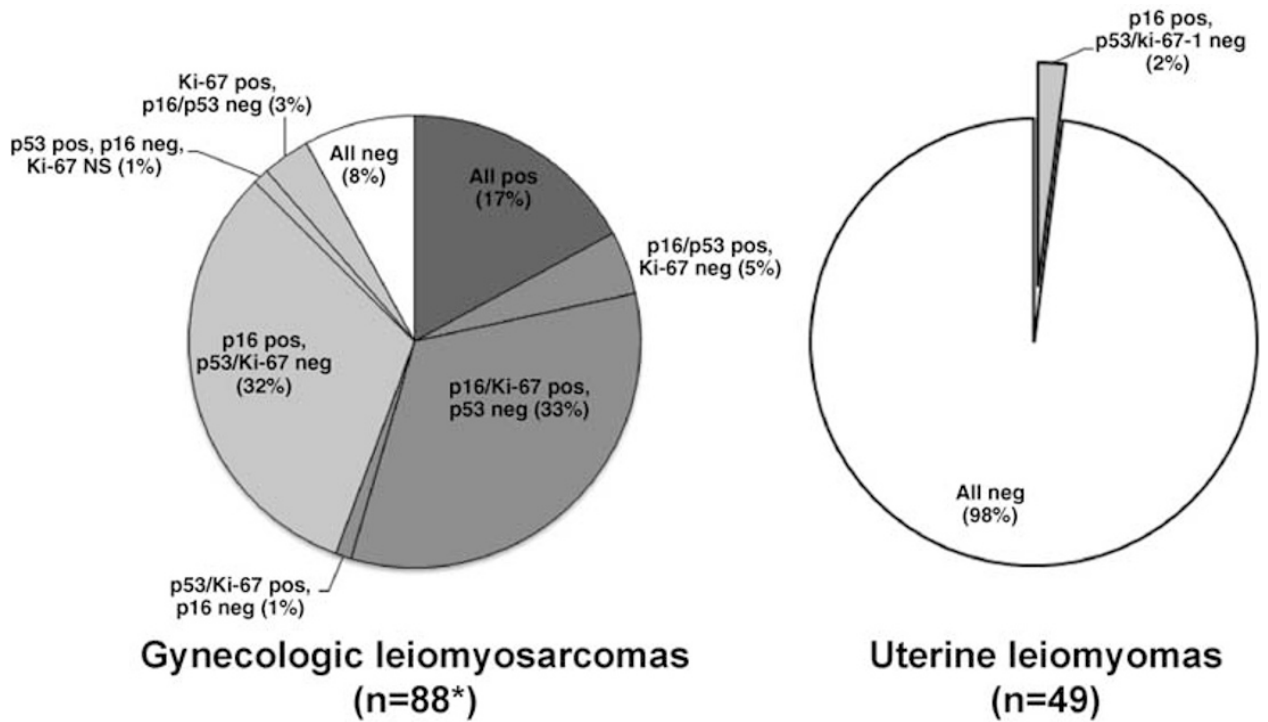

\section{Uterine leiomyomas \\ $(n=49)$}

Figure 6 Summary of the immunohistochemical staining results in gynecologic leiomyosarcomas and uterine leiomyomas (pos, positive; neg, negative; NS, nonscorable; *, number of scorable cases).

patients. Setting a cutoff at 5\% may allow for better discrimination between gynecologic and nongynecologic leiomyosarcomas. In support of this notion, we have observed from unpublished gene expression profiling study that 9 of 17 $(53 \%)$ gynecologic leiomyosarcomas but only 1 of 26 (4\%) nongynecologic leiomyosarcomas show significant ESR1 expression levels (unpublished data).

As to WT1, 8\% of the gynecologic leiomyosarcomas and $0 \%$ of nongynecologic leiomyosarcomas showed positive nuclear staining whereas $55 \%$ of gynecologic leiomyosarcomas and $52 \%$ of nongynecologic leiomyosarcomas showed positive cytoplasmic staining. This finding is different from that reported by Deavers et $a 1^{15}$ both qualitatively and quantitatively. Using the same antibody targeting WT1 (Dako clone 6F-H2), Deavers et al ${ }^{15}$ reported, in a series of 20 cases, positive nuclear WT1 staining in $60 \%$ of gynecologic leiomyosarcomas and in $0 \%$ of nongynecologic leiomyosarcomas. Two other previous studies have also assessed immunohistochemical expression of WT1 in gynecologic and nongynecologic leiomyosarcomas using the same anti-WT1 antibody. ${ }^{13,14}$ The findings from these two studies are more comparable to our current findings as they reported predominantly cytoplasmic pattern of immunostaining in $64-76 \%$ of gynecologic leiomyosarcomas with one study reporting predominantly cytoplasmic WT1 immunostaining in 58\% of nongynecologic leiomyosarcomas. The reason underlying these discrepant findings is unclear though Deavers et al appeared to have used a different secondary antibody detection system based on the abstract that was published. ${ }^{33}$ Regardless of the source of this discrepancy our data indicate that
WT1 is a less useful marker than ER in determining site of origin for smooth muscle tumors.

\section{Adjunct Markers to Differentiate between Malignant and Benign Gynecologic Smooth Muscle Tumors}

Three immunomarkers-p16, Ki-67 and p53-were examined for their utility in differentiating between malignant and benign primary gynecologic smooth muscle tumors. Similar to the reported frequency of p16 positivity in $84 \%$ of gynecologic leiomyosarcomas (101 cases from 5 studies), we now observed p16 positivity in $87 \%$ of gynecologic leiomyosarcomas in our series. Only $2 \%$ of uterine leiomyomas from our series exhibited p16 positivity (positive staining in $\geq 50 \%$ of tumor cells), whereas 11 of $108(10 \%)$ combined cases from 5 published studies of uterine leiomyoma showed variable degree of positive p16 staining. ${ }^{17-20,31}$ Of these 11 positive cases, 8 originated from studies in which a lower cutoff for p16 positivity (25 and $33 \%$ ) was used $^{17-19}$ and the 3 remaining positive cases came from a study that considered any strong immunostaining of tumor cells to be positive. ${ }^{20}$ In general, all of the studies reported the pattern of p16 to be typically focal and weak in uterine leiomyomas, in contrast to the more diffuse and intense pattern of staining seen in leiomyosarcomas. It would appear based on our current finding that the use of a higher threshold value (ie 50\%) for p16 can improve the specificity of p16 without significant compromise to its sensitivity.

Positive p53 immunostaining was found in 29\% of gynecologic leiomyosarcomas and $0 \%$ of uterine leiomyomas in our current series based on a cutoff of 
Table 3 Summary of the immunostaining results from the current study and previously published studies

\begin{tabular}{|c|c|c|c|c|c|c|}
\hline Studies & $\begin{array}{c}\text { Gynecologic } \\
\text { leiomyosarcomas }\end{array}$ & $\begin{array}{l}\text { Nongynecologic } \\
\text { leiomyosarcomas }\end{array}$ & $\begin{array}{l}\text { Smooth muscle } \\
\text { tumors of uncertain } \\
\text { malignant potential }\end{array}$ & $\begin{array}{c}\text { Uterine leiomyomas, } \\
\text { variant }^{\mathrm{a}}\end{array}$ & $\begin{array}{c}\text { Uterine } \\
\text { leiomyomas }\end{array}$ & $\begin{array}{c}\text { Criteria for } \\
\text { positivity }\end{array}$ \\
\hline \multicolumn{7}{|l|}{ ER staining } \\
\hline Ip et $a l^{39}$ & & & 15 of 15 & & & All > $10 \%$ \\
\hline Akhan et $a l^{4}$ & 5 of 19 & & & & & $>10 \%$ \\
\hline Leitao et $a l^{9}$ & 10 of 25 & & & & 15 of 19 & NA \\
\hline Kelley et $a l^{8}$ & 13 of 15 & 4 of 16 & & & & $>1 \%$ \\
\hline Bodner et $a l^{18}$ & 12 of 21 & & 13 of 24 & & 24 of 26 & $>10 \%$ \\
\hline Bodner et $a l^{18}$ & 12 of 21 & & & & & $>10 \%$ \\
\hline Zhai et $a l^{11}$ & 5 of 14 & & 5 of 8 & 18 of 18 & 20 of 20 & $>5 \%$ \\
\hline Rao et $a l^{10}$ & 10 of 14 & 1 of 16 & & & 5 of 5 & $>5 \%$ \\
\hline Combined published data & 67 of $129(52 \%)$ & 5 of $32(16 \%)$ & 33 of $47(70 \%)$ & 18 of $18(100 \%)$ & 64 of $70(91 \%)$ & \\
\hline Data from current study & 51 of $102(50 \%)$ & 4 of $140(3 \%)$ & & 3 of $3(100 \%)$ & 46 of $46(100 \%)$ & $\geq 5 \%$ \\
\hline Overall combined data & 118 of $231(51 \%)$ & 9 of $172(5 \%)$ & 33 of $47(70 \%)$ & 21 of $21(100 \%)$ & 110 of $116(95 \%)$ & \\
\hline \multicolumn{7}{|l|}{ WT1 staining } \\
\hline Bing et $a l^{13}$ & 16 of 25 & 14 of 24 & & & & $>5 \%$ \\
\hline Deavers et $a l^{15}$ & 12 of 20 & 0 of 22 & 3 of 3 & 3 of 3 & 16 of 16 & Any \\
\hline Coosemans et al ${ }^{14}$ & 29 of 38 & & & & & Any \\
\hline Agoff et $a l^{40}$ & & & & 9 of 9 & & NA \\
\hline Combined published data & 57 of $83(69 \%)$ & 14 of $46(30 \%)$ & 3 of $3(100 \%)$ & 12 of $12(100 \%)$ & 16 of $16(100 \%)$ & \\
\hline Data from current study & 54 of $98(55 \%)$ & 68 of $131(52 \%)$ & & 3 of $3(100 \%)$ & 42 of $46(91 \%)$ & $\geq 5 \%$ \\
\hline Overall combined data & 111 of $181(61 \%)$ & 82 of $177(46 \%)$ & 3 of $3(100 \%)$ & 15 of $15(100 \%)$ & 58 of $62(94 \%)$ & \\
\hline \multicolumn{7}{|l|}{ p53 staining } \\
\hline Ip et $a l^{39}$ & & & 2 of 15 & & & $>66 \%$ \\
\hline Chen et $a l^{19}$ & 32 of 35 & & 1 of 2 & 9 of 28 & 0 of 35 & $>$ moderate \\
\hline O’Neill et $a l^{31}$ & 8 of 22 & & 0 of 4 & 2 of 27 & 0 of 10 & $>25 \%$ \\
\hline Anderson et $a l^{34}$ & 12 of 25 & & & & 0 of 19 & NA \\
\hline Akhan et $a l^{4}$ & 4 of 19 & & & & & $>25 \%$ \\
\hline Mittal et $a l^{23}$ & 5 of 12 & & 0 of 7 & 0 of 15 & & $\geq 15 \%$ \\
\hline Layfield et $a l^{37}$ & 4 of 9 & & & & 0 of 15 & $>5 \%$ \\
\hline Zhai et $a l^{11}$ & 7 of 14 & & 0 of 8 & 0 of 18 & 0 of 20 & $>5 \%$ \\
\hline Blom et $a l^{35}$ & 13 of 49 & & & & & $>10 \%$ \\
\hline Hall et $a 2^{21}$ & 3 of 23 & & & & 0 of 20 & NA \\
\hline De Vos et $a l^{36}$ & 3 of 8 & & & & 0 of 8 & NA \\
\hline Amada et $a l^{27}$ & 8 of 24 & & & 0 of 25 & & Any \\
\hline Niemann et $a l^{38}$ & 16 of 34 & & 1 of 6 & & 0 of 18 & $>20 \%$ \\
\hline Jeffers et $a l^{29}$ & 13 of 23 & & 6 of 10 & & 1 of 18 & NA \\
\hline Konomoto et $a l^{41}$ & & 8 of 37 & & & & Any \\
\hline O'Reilly et $a l^{32}$ & & 19 of 44 & & & & Any \\
\hline Combined published data & 128 of $297(43 \%)$ & 27 of $81(33 \%)$ & 10 of $52(20 \%)$ & 11 of $113(10 \%)$ & 1 of $163(0.6 \%)$ & \\
\hline Data from current study & 25 of $87(29 \%)$ & 21 of $96(22 \%)$ & & 0 of $3(0 \%)$ & 0 of $46(0 \%)$ & \\
\hline Overall combined data & 153 of $384(40 \%)$ & 48 of $177(27 \%)$ & 10 of $52(20 \%)$ & 11 of $116(9 \%)$ & 1 of $209(0.5 \%)$ & \\
\hline \multicolumn{7}{|l|}{ p16 staining } \\
\hline Ip et $a l^{39}$ & & & 2 of 15 & & & $>66 \%$ \\
\hline Atkins et $a l^{17}$ & 11 of 15 & & 3 of 8 & & 0 of 23 & $>33 \%$ \\
\hline Gannon et $a l^{20}$ & 8 of 8 & & & 7 of 31 & 3 of 14 & $\begin{array}{c}\text { Any moderate/ } \\
\text { strong }\end{array}$ \\
\hline Chen et $a l^{19}$ & 35 of 35 & & 2 of 2 & 18 of 28 & 5 of 35 & $>25 \%$ \\
\hline O’Neill et $a l^{31}$ & 19 of 22 & & 1 of 4 & 7 of 27 & 0 of 10 & $>50 \%$ \\
\hline Bodner-Adler et $a l^{18}$ & 12 of 21 & & 5 of 24 & & 3 of 26 & $>33 \%$ \\
\hline Combined published data & 85 of $101(84 \%)$ & & 13 of $53(25 \%)$ & 32 of $86(37 \%)$ & 11 of $108(10 \%)$ & \\
\hline Data from current study & 77 of $89(87 \%)$ & & & o of 3 & 1 of $45(2 \%)$ & $\geq 50 \%$ \\
\hline Overall combined data & 162 of $190(85 \%)$ & & 13 of $53(25 \%)$ & 32 of $89(36 \%)$ & 12 of $153(8 \%)$ & \\
\hline \multicolumn{7}{|l|}{ Ki-67 labeling index } \\
\hline Ip et $a l^{39}$ & & & 1 of 15 & & & $>10 \%$ \\
\hline Chen et $a l^{19}$ & 29 of 35 & & 2 of 2 & 7 of 28 & 0 of 35 & $>10 \%$ \\
\hline O’Neill et $a l^{31}$ & 20 of 22 & & 2 of 4 & 14 of 27 & 4 of 10 & $>10 \%$ \\
\hline Akhan et $a l^{4}$ & 14 of 19 & & & & & $>10 \%$ \\
\hline Mayerhofer et $a l^{22}$ & 10 of 20 & & 0 of 22 & & 2 of 25 & $>10 \%$ \\
\hline Mayerhofer et $a l^{22}$ & 10 of 20 & & & & & $>10 \%$ \\
\hline Mittal et $a l^{23}$ & 11 of 12 & & 0 of 7 & 0 of 15 & & $>10 \%$ \\
\hline Chou et $a l^{28}$ & 3 of 6 & & & & 0 of 44 & $>10 \%$ \\
\hline Amada et $a l^{27}$ & 13 of 24 & & & 0 of 25 & & $>10 \%$ \\
\hline O'Reilly $^{32}$ & & 29 of 44 & & & & Any \\
\hline Combined published data & 110 of $158(70 \%)$ & 29 of $44(66 \%)$ & 5 of $50(10 \%)$ & 21 of $95(22 \%)$ & 6 of $114(5 \%)$ & \\
\hline Data from current study & 51 of $78(65 \%)$ & 56 of $79(71 \%)$ & & 0 of $3(0 \%)$ & 0 of $46(0 \%)$ & $>10 \%$ \\
\hline Overall combined data & 161 of $236(68 \%)$ & 85 of $123(69 \%)$ & 5 of $50(10 \%)$ & 21 of $98(21 \%)$ & 6 of $160(4 \%)$ & \\
\hline
\end{tabular}

NA: not available.

${ }^{\mathrm{a}}$ The uterine leiomyoma variant includes cellular leiomyoma and symplastic leiomyoma.

Values in italics are used for summated values. 
$50 \%$ nuclear staining. A large number of studies have previously examined the pattern of p53 immunostaining in gynecologic smooth muscle tumors and cumulatively across 297 cases, p53 positivity was observed in $43 \%$ of gynecologic leiomyosarcomas and less than $1 \%$ of uterine leiomyomas. ${ }^{4,7,11,19,21,23,27,29,31,34-38}$ The lower frequency of p53 positivity seen in our study may be attributed to the significantly lower cutoff value used by several of these earlier studies, whereas the two previous studies that used a more comparable cutoff value of $25 \%$ showed a similar rate of p53 positivity ranging from 21 to $36 \%$ of the cases. ${ }^{4,31}$ Even though the sensitivity of p53 is poor for detecting malignant gynecologic smooth muscle tumors, its specificity appears to be exceptional based on all available data $(>99 \%)$.

In this study, the percentage of Ki-67-positive nuclear staining was evaluated both visually and by automated morphometric analysis. The results were highly concordant between these two analytic methods, with about two-thirds of primary gynecologic leiomyosarcomas and $0 \%$ of uterine leiomyomas showing $>10 \%$ positive nuclear staining. In comparison, $70 \%$ of previously reported gynecologic leiomyosarcomas (158 cases from 8 studies) and $5 \%$ of previously reported conventional uterine leiomyomas (114 cases from 6 studies) showed $>10 \%$ Ki-67 labeling index by visual method of analysis. $^{4,7,11,19,22,23,27-32}$ It is unclear whether any of these uterine leiomyomas with elevated Ki-67 labeling index exhibited increased mitotic index.

A limitation of our current study is the paucity of variant uterine leiomyomas and lack of smooth muscle tumors of uncertain malignant potential examined. Only one symplastic and two cellular uterine leiomyomas were included in our current series. Although all three cases demonstrated negative staining for p16 and p53, and were all associated with a low Ki-67 proliferation index, it is certainly of insufficient sample size for meaningful assessment. Our review of the literature showed that a higher percentage of variant uterine leiomyomas (inclusive of symplastic uterine leiomyoma and cellular uterine leiomyoma) and smooth muscle tumors of uncertain malignant potential demonstrate positive p16 or p53 staining, though as noted earlier, these studies used a lower threshold cutoff value for positivity. In addition, 11 and $22 \%$ of smooth muscle tumors of uncertain malignant potential and variant uterine leiomyomas, respectively, appear to exhibit greater than 10\% Ki-67 nuclear staining by tumor cells. ${ }^{19,22,23,27,31}$ However, most of these earlier studies lacked clinical follow-up data necessary for correlation with outcome. Intriguingly, recent studies have shown a tendency for smooth muscle tumors of uncertain malignant potential that behave in a malignant manner, with local or systemic recur- rences to exhibit diffuse p16 and/or p53 immunostaining. ${ }^{17,39}$ Most recently, Ip et $a l^{39}$ observed the presence of diffuse p16 and p53 positivity in 2 of 16 smooth muscle tumors of uncertain malignant potential examined, both of which developed local recurrence within 5 years after the initial surgery and were the only cases in which the disease recurred in that series. Further studies are needed to evaluate the utility of p16, p53 and Ki-67 in predicting the clinical behavior of these diagnostically challenging gynecologic smooth muscle tumors. Another limitation of the study is that we only evaluated the staining patterns of tissue cores containing representative areas of the tumors and did not assess for potential intratumoral variability on whole sections for each cases. Although the current study helps to establish the staining patterns between benign and malignant smooth muscle tumors, future study looking at staining patterns on whole tissue sections will be needed, particularly focussing on diagnostically challenging cases.

Although not the focus of our current study, we analyzed the ability of our markers to predict outcome in cases diagnosed as leiomyosarcoma. We did not find a significant association between survival and the Ki-67 proliferation index for gynecologic leiomyosarcomas in our current series. In contrast, two studies have previously reported improved survival in patients with gynecologic leiomyosarcomas that show lower proliferation index (using 10 and $30 \%$ cutoffs). ${ }^{4,22}$ No significant association with disease-specific survival was found in the current series for p53 and ER positivity in gynecologic leiomyosarcomas either.

In summary, we have shown the utility of p16, p53 and Ki-67 in differentiating between malignant and benign tumors in a large series of primary gynecologic smooth muscle tumors. We have also shown the specificity of ER positivity for leiomyosarcomas of gynecologic origin in a large series of primary/recurrent gynecologic and nongynecologic leiomyosarcomas. These findings can help pathologists when faced with these challenging diagnostic scenarios.

\section{Acknowledgements}

This study was supported by the British Columbia Cancer Agency's Musculoskeletal Tumor Group. Work at Stanford University was supported by the National Institute of Health (CA112270), the National Leiomyosarcoma Foundation and the Leiomyosarcoma Direct Research Foundation.

\section{Disclosure/conflict of interest}

The authors declare no conflict of interest. 


\section{References}

1 Miettinen M, Fetsch JF. Evaluation of biological potential of smooth muscle tumours. Histopathology 2006;48:97-105.

2 Giuntoli 2nd RL, Bristow RE. Uterine leiomyosarcoma: present management. Curr Opin Oncol 2004;16: 324-327.

3 Mankin HJ, Hornicek FJ. Diagnosis, classification, and management of soft tissue sarcomas. Cancer Control 2005;12:5-21.

4 Akhan SE, Yavuz E, Tecer A, et al. The expression of Ki-67, p53, estrogen and progesterone receptors affecting survival in uterine leiomyosarcomas. A clinicopathologic study. Gynecol Oncol 2005;99:36-42.

5 Bodner $\mathrm{K}$, Bodner-Adler $\mathrm{B}$, Kimberger $\mathrm{O}$, et al. Estrogen and progesterone receptor expression in patients with uterine leiomyosarcoma and correlation with different clinicopathological parameters. Anticancer Res 2003;23:729-732.

6 Bodner $\mathrm{K}$, Bodner-Adler $\mathrm{B}$, Kimberger $\mathrm{O}$, et al. Estrogen and progesterone receptor expression in patients with uterine smooth muscle tumors. Fertil Steril 2004;81:1062-1066.

7 Gokaslan H, Turkeri L, Kavak ZN, et al. Differential diagnosis of smooth muscle tumors utilizing p53, pTEN and Ki-67 expression with estrogen and progesterone receptors. Gynecol Obstet Invest 2005;59:36-40.

8 Kelley TW, Borden EC, Goldblum JR. Estrogen and progesterone receptor expression in uterine and extrauterine leiomyosarcomas: an immunohistochemical study. Appl Immunohistochem Mol Morphol 2004;12: 338-341.

9 Leitao MM, Soslow RA, Nonaka D, et al. Tissue microarray immunohistochemical expression of estrogen, progesterone, and androgen receptors in uterine leiomyomata and leiomyosarcoma. Cancer 2004;101: 1455-1462.

10 Rao UN, Finkelstein SD, Jones MW. Comparative immunohistochemical and molecular analysis of uterine and extrauterine leiomyosarcomas. Mod Pathol 1999;12:1001-1009.

11 Zhai YL, Kobayashi Y, Mori A, et al. Expression of steroid receptors, Ki-67, and p53 in uterine leiomyosarcomas. Int J Gynecol Pathol 1999;18:20-28.

12 Jakimiuk AJ, Bogusiewicz M, Tarkowski R, et al. Estrogen receptor alpha and beta expression in uterine leiomyomas from premenopausal women. Fertil Steril 2004;82:1244-1249.

13 Bing Z, Pasha TL, Acs G, et al. Cytoplasmic overexpression of WT-1 in gastrointestinal stromal tumor and other soft tissue tumors. Appl Immunohistochem Mol Morphol 2008;16:316-321.

14 Coosemans A, Nik SA, Caluwaerts S, et al. Upregulation of Wilms' tumour gene 1 (WT1) in uterine sarcomas. Eur J Cancer 2007;43:1630-1637.

15 Deavers MT. Immunohistochemistry in gynecologic pathology. Arch Pathol Lab Med 2008;132:175-180.

16 Giuntoli 2nd RL, Gostout BS, DiMarco CS, et al. Diagnostic criteria for uterine smooth muscle tumors: leiomyoma variants associated with malignant behavior. J Reprod Med 2007;52:1001-1010.

17 Atkins KA, Arronte N, Darus CJ, et al. The use of p16 in enhancing the histologic classification of uterine smooth muscle tumors. Am J Surg Pathol 2008;32: 98-102.
18 Bodner-Adler B, Bodner K, Czerwenka K, et al. Expression of p16 protein in patients with uterine smooth muscle tumors: an immunohistochemical analysis. Gynecol Oncol 2005;96:62-66.

19 Chen L, Yang B. Immunohistochemical analysis of p16, p53, and Ki-67 expression in uterine smooth muscle tumors. Int J Gynecol Pathol 2008;27:326-332.

20 Gannon BR, Manduch M, Childs TJ. Differential immunoreactivity of p16 in leiomyosarcomas and leiomyoma variants. Int J Gynecol Pathol 2008;27: 68-73.

21 Hall KL, Teneriello MG, Taylor RR, et al. Analysis of Ki-ras, p53, and MDM2 genes in uterine leiomyomas and leiomyosarcomas. Gynecol Oncol 1997;65: 330-335.

22 Mayerhofer K, Lozanov P, Bodner K, et al. Ki-67 expression in patients with uterine leiomyomas, uterine smooth muscle tumors of uncertain malignant potential (STUMP) and uterine leiomyosarcomas (LMS). Acta Obstet Gynecol Scand 2004;83: 1085-1088.

23 Mittal K, Demopoulos RI. MIB-1 (Ki-67), p53, estrogen receptor, and progesterone receptor expression in uterine smooth muscle tumors. Hum Pathol 2001;32: 984-987.

24 Lee CH, Espinosa I, Vrijaldenhoven S, et al. Prognostic significance of macrophage infiltration in leiomyosarcomas. Clin Cancer Res 2008;14:1423-1430.

25 Espinosa I, Beck AH, Lee CH, et al. Coordinate expression of colony-stimulating factor-1 and colonystimulating factor-1-related proteins is associated with poor prognosis in gynecological and nongynecological leiomyosarcoma. Am J Pathol 2009;174:2347-2356.

26 Steigen SE, Straume B, Turbin D, et al. Clinicopathologic factors and nuclear morphometry as independent prognosticators in KIT-positive gastrointestinal stromal tumors. J Histochem Cytochem 2008;56:139-145.

27 Amada S, Nakano H, Tsuneyoshi M. Leiomyosarcoma versus bizarre and cellular leiomyomas of the uterus: a comparative study based on the MIB-1 and proliferating cell nuclear antigen indices, p53 expression, DNA flow cytometry, and muscle specific actins. Int J Gynecol Pathol 1995;14:134-142.

28 Chou CY, Huang SC, Tsai YC, et al. Uterine leiomyosarcoma has deregulated cell proliferation, but not increased microvessel density compared with uterine leiomyoma. Gynecol Oncol 1997;65:225-231.

29 Jeffers MD, Oakes SJ, Richmond JA, et al. Proliferation, ploidy and prognosis in uterine smooth muscle tumours. Histopathology 1996;29:217-223.

30 Mayerhofer K, Lozanov P, Bodner K, et al. Ki-67 and vascular endothelial growth factor expression in uterine leiomyosarcoma. Gynecol Oncol 2004;92: 175-179.

31 O’Neill CJ, McBride HA, Connolly LE, et al. Uterine leiomyosarcomas are characterized by high p16, p53 and MIB1 expression in comparison with usual leiomyomas, leiomyoma variants and smooth muscle tumours of uncertain malignant potential. Histopathology 2007;50:851-858.

32 O'Reilly PE, Raab SS, Niemann TH, et al. p53, proliferating cell nuclear antigen, and Ki-67 expression in extrauterine leiomyosarcomas. Mod Pathol 1997;10:91-97.

33 Deavers MT, Silva EG, Euscher ED, et al. WT1 expression may differentiate mullerian from non-mullerian smooth muscle tumors. Mod Pathol 2006;19:176A. 
34 Anderson SE, Nonaka D, Chuai S, et al. p53, epidermal growth factor, and platelet-derived growth factor in uterine leiomyosarcoma and leiomyomas. Int J Gynecol Cancer 2006;16:849-853.

35 Blom R, Guerrieri C, Stal O, et al. Leiomyosarcoma of the uterus: a clinicopathologic, DNA flow cytometric, p53, and mdm-2 analysis of 49 cases. Gynecol Oncol 1998;68:54-61.

36 de Vos S, Wilczynski SP, Fleischhacker M, et al. p53 alterations in uterine leiomyosarcomas versus leiomyomas. Gynecol Oncol 1994;54:205-208.

37 Layfield LJ, Liu K, Dodge R, et al. Uterine smooth muscle tumors: utility of classification by proliferation, ploidy, and prognostic markers versus traditional histopathology. Arch Pathol Lab Med 2000;124:221-227.
38 Niemann TH, Raab SS, Lenel JC, et al. p53 protein overexpression in smooth muscle tumors of the uterus. Hum Pathol 1995;26:375-379.

39 Ip PP, Cheung AN, Clement PB. Uterine smooth muscle tumors of uncertain malignant potential (STUMP): a clinicopathologic analysis of 16 cases. Am J Surg Pathol 2009;33:992-1005.

40 Agoff SN, Grieco VS, Garcia R, Gown AM. Immunohistochemical distinction of endometrial stromal sarcoma and cellular leiomyoma. Appl Immunohistochem Mol Morphol 2001;9:164-169.

41 Konomoto T, Fukuda T, Hayashi K, et al. Leiomyosarcoma in soft tissue: examination of p53 status and cell proliferating factors in different locations. Hum Pathol 1998;29:74-81.

Supplementary Information accompanies the paper on Modern Pathology website (http://www.nature.com/ modpathol) 